\title{
TITLE:
}

\section{3-D magnetohydrodynamic field computation of supersonic duct flow of weakly ionized plasma}

$\operatorname{AUTHOR}(S)$ :

Matsuo, T; Tadamatsu, A; Shimasaki, M

CITATION:

Matsuo, T ... [et al]. 3-D magnetohydrodynamic field computation of supersonic duct flow of weakly ionized plasma. IEEE TRANSACTIONS ON MAGNETICS 2003, 39(3): 1444-1447

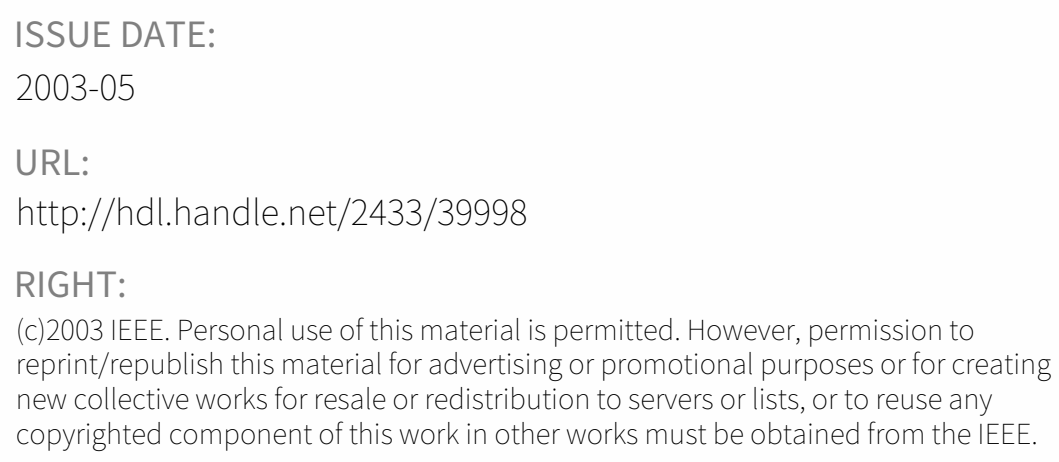




\title{
3-D Magnetohydrodynamic Field Computation of Supersonic Duct Flow of Weakly Ionized Plasma
}

\author{
Tetsuji Matsuo, Member, IEEE, Akihiro Tadamatsu, and Masaaki Shimasaki, Member, IEEE
}

\begin{abstract}
Supersonic magnetohydrodynamic field analysis is carried out for energy conversion using a weakly ionized plasma. The magnetic field induced by a generated electric current is taken into account. A three-dimensional simulation shows that the Lorentz force induces a shock wave and boundary-layer separation in the plasma duct flow.
\end{abstract}

Index Terms-Boundary-layer separation, induced magnetic field, magnetohydrodynamics (MHD), shock wave.

\section{INTRODUCTION}

$\mathbf{H}$ IGH-SPEED flows of weakly ionized plasma have a wide range of application. These include magnetohydrodynamic (MHD) generator/accelerators and MHD hypersonic flow control. The analysis of such high-speed plasma flows results in the coupled problem of electromagnetics with high-power density and gasdynamics with shock waves and boundary-layer separation.

Analyses of MHD generators usually ignore the magnetic field induced by the generated electric current because the magnetic Reynolds number of typical MHD generators is much less than one. Some MHD generators, however, have relatively large induced magnetic fields because of their high-electric power density, where the magnetic Reynolds number becomes of the order of 0.1 . The application to MHD hypersonic flow control also requires analysis taking into account the induced magnetic field.

This paper carries out a three-dimensional (3-D) MHD field analysis taking into account the induced magnetic field for a supersonic plasma flow with a power density of more than 300 $\mathrm{MW} / \mathrm{m}^{3}$.

\section{ANALYZED MHD FLOW}

A plasma flow of one of the generator ducts of the Pamir-3U MHD power system [1], [2] is analyzed in this paper. Pamir-3U is a portable, self-excited pulsed-MHD power system with a maximum power output of $15 \mathrm{MW}$.

Fig. 1 shows the generator duct, which has a pair of continuous electrodes (a cathode and an anode). The $x, y$, and $z$ directions in the present analysis represent the directions of the core flow, the Faraday current induced and the applied magnetic field, respectively. The half domain of the duct $(z \geq 0)$

Manuscript received June 18, 2002.

T. Matsuo and M. Shimasaki are with the Graduate School of Engineering, Kyoto University, Kyoto 606-8501, Japan (e-mail: tmatsuo@kuee.kyotou.ac.jp; simasaki@kuee.kyoto-u.ac.jp).

A. Tadamatsu was with the Graduate School of Engineering, Kyoto University, Kyoto 606-8501, Japan. He is now with NTT DoCoMo, Inc., Tokyo 105-0001, Japan

Digital Object Identifier 10.1109/TMAG.2003.810169

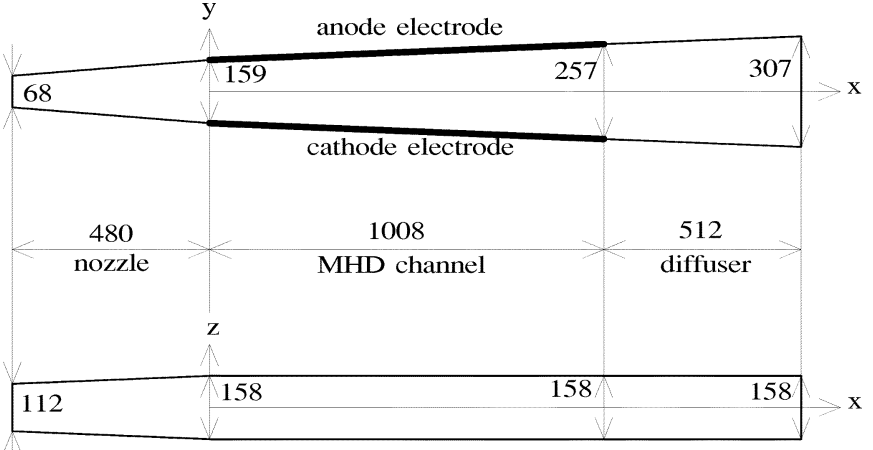

Fig. 1. Generator duct (unit: millimeters).

is analyzed on account of its symmetry. The generator duct is nonuniformly divided into $244 \times 40 \times 16$ cells.

The working fluid in the generator duct is a weakly ionized plasma of solid-propellant combustion gas with cesium seed. The applied magnetic flux density is 3-5 T. The electrical conductivity of the gas is $70-120 \mathrm{~S} / \mathrm{m}$ with a Hall parameter of $0.4-1$. The gas velocity is more than $1 \mathrm{~km} / \mathrm{s}$.

The weakly ionized plasma in the generator duct is analyzed under the following assumptions: 1) the induced magnetic field is smaller than the applied magnetic field since the magnetic Reynolds number is less than one; 2) the displacement current is much smaller than the conduction current and is negligible; and 3 ) the time variation in the magnetic flux density is negligible.

\section{Method OF MHD FIELD ANALYSIS}

\section{A. Electromagnetic Field Analysis}

The electromagnetic field in the plasma flow is governed by

$$
\begin{aligned}
\operatorname{rot}\left(\frac{B}{\mu}\right) & =\boldsymbol{J}, \quad \operatorname{div} \boldsymbol{B}=0 \\
\operatorname{rot} \boldsymbol{E} & =0, \quad \operatorname{div} \boldsymbol{J}=0 \\
\boldsymbol{J} & =\boldsymbol{\Sigma}(\boldsymbol{B})(\boldsymbol{E}+\boldsymbol{u} \times \boldsymbol{B})
\end{aligned}
$$

where $\boldsymbol{B}=\left(B_{x}, B_{y}, B_{z}\right)$ is the magnetic flux density, $\boldsymbol{J}=$ $\left(J_{x}, J_{y}, J_{z}\right)$ is the electric current density, $\boldsymbol{E}=\left(E_{x}, E_{y}, E_{z}\right)$ is the electric field, $\boldsymbol{u}=\left(u_{x}, u_{y}, u_{z}\right)$ is the gas velocity, and $\boldsymbol{\Sigma}(\boldsymbol{B})$ is the conductivity tensor given by

$$
\boldsymbol{\Sigma}=\sigma\left[\begin{array}{ccc}
1 & m B_{z} & -m B_{y} \\
-m B_{z} & 1 & m B_{x} \\
m B_{y} & -m B_{x} & 1
\end{array}\right]^{-1}
$$

where $\sigma$ is the electrical conductivity, $m=\beta /\|B\|$, and $\beta$ is the Hall parameter. The dependences of $\sigma$ and $\beta$ on the gas temperature and pressure are given in [5]. The permeability $\mu$ is set at $4 \pi \times 10^{-7}(\mathrm{H} / \mathrm{m})$. 
The magnetic flux density $\boldsymbol{B}$ consists of the applied magnetic flux density $\left(\boldsymbol{B}_{a}\right)$ and the induced magnetic flux density $\left(\boldsymbol{B}_{i}\right)$. It is assumed that $\boldsymbol{B}_{a}$ is known and that $\left\|\boldsymbol{B}_{a}\right\| \gg\left\|\boldsymbol{B}_{i}\right\|$. The fields $\boldsymbol{B}_{a}$ and $\boldsymbol{B}_{i}$ satisfy (5) and (6) in the generator duct, respectively.

$$
\begin{aligned}
\operatorname{rot} \boldsymbol{B}_{a} & =0 \\
\operatorname{rot} \boldsymbol{B}_{i} & =\mu \boldsymbol{J}
\end{aligned}
$$

It is assumed that the unknown field $\boldsymbol{B}_{\boldsymbol{i}}$ is approximated by $\boldsymbol{B}_{i 0}$. First, by setting $\boldsymbol{B}=\boldsymbol{B}_{0}=\boldsymbol{B}_{a}+\boldsymbol{B}_{i 0}$, (2) and (3) lead to

$$
\operatorname{div} \boldsymbol{J}_{0}=\operatorname{div}\left\{\boldsymbol{\Sigma}\left(\boldsymbol{B}_{0}\right)\left(-\operatorname{grad} \phi_{0}+\boldsymbol{u} \times \boldsymbol{B}_{0}\right)\right\}=0
$$

where $\phi_{0}$ is the electric potential satisfying

$$
-\operatorname{grad} \phi_{0}=\boldsymbol{E}_{0}
$$

and $\boldsymbol{J}_{0}$ and $\boldsymbol{E}_{0}$ are $\boldsymbol{J}$ and $\boldsymbol{E}$ when $\boldsymbol{B}=\boldsymbol{B}_{0}$, respectively. It is assumed that $u$ is known from the gasdynamical analysis. Equation (7) is solved for $\phi_{0}$ by the Galerkin finite-element method (FEM) to obtain $\boldsymbol{J}_{0}$ and $\boldsymbol{E}_{0}$ from (3) and (8).

Next, $\boldsymbol{J}_{0}$ is used to determine $\boldsymbol{B}_{i}$ from (6) as

$$
\operatorname{rot}\left(\operatorname{rot} \boldsymbol{A}_{i}\right)=\mu \boldsymbol{J}_{0}
$$

where $\boldsymbol{A}_{i}$ is the magnetic vector potential that satisfies

$$
\operatorname{rot} A_{i}=B_{i} \text {. }
$$

The solution to (9) for $\boldsymbol{A}_{\boldsymbol{i}}$ by the Galerkin FEM gives a more accurate $\boldsymbol{B}_{i}$.

After correcting $\boldsymbol{B}\left(=\boldsymbol{B}_{a}+\boldsymbol{B}_{i}\right), \boldsymbol{E}$ and $\boldsymbol{J}$ are recalculated from (11)

$$
\begin{aligned}
\operatorname{div} \boldsymbol{J}=\operatorname{div}[ & {\left[\left(\boldsymbol{B}_{a}+\boldsymbol{B}_{i}\right)\right.} \\
& \left.\cdot\left\{-\operatorname{grad} \phi+\boldsymbol{u} \times\left(\boldsymbol{B}_{a}+\boldsymbol{B}_{i}\right)\right\}\right]=0
\end{aligned}
$$

where $\phi$ satisfies

$$
-\operatorname{grad} \phi=\boldsymbol{E} .
$$

The solution to (11) for $\phi$ by the Galerkin FEM gives $\boldsymbol{J}$ and $\boldsymbol{E}$ from (3) and (12).

\section{B. Gasdynamical Analysis}

The basic equations used for gasdynamics are the time-dependent 3-D compressible Navier-Stokes equations

$$
\begin{aligned}
\frac{\partial \rho}{\partial t}+\nabla \cdot(\rho \mathbf{u}) & =0 \\
\frac{\partial(\rho u)}{\partial t}+\nabla \cdot(\rho \boldsymbol{u u}) & =-\nabla p+\nabla \boldsymbol{\tau}+\boldsymbol{J} \times \boldsymbol{B} \\
\frac{\partial \varepsilon}{\partial t}+\nabla \cdot\{(\varepsilon+p) \boldsymbol{u}\} & =\nabla \cdot(\boldsymbol{\tau} \boldsymbol{u}-\boldsymbol{q})+\boldsymbol{J} \cdot \boldsymbol{E}
\end{aligned}
$$

where $\rho$ is the mass density, $\boldsymbol{u}$ is the velocity, $p$ is the pressure, $\varepsilon=\rho\left(e_{0}+\|\boldsymbol{u}\|^{2} / 2\right)$, where $e_{0}$ is the gas internal energy; $\boldsymbol{\tau}$ is the shear stress tensor and $\boldsymbol{q}$ is the heat flux. The mixinglength turbulence model [3], [4] is used to describe the turbulent viscosity. The thermodynamical properties of the working fluid are given by [5].

The Navier-Stokes equations (13)-(15) are solved by the Harten-Yee upwind explicit total variation diminishing (TVD) scheme [6].

The multitime-step method [7] is applied to the gasdynamical analysis in order to give efficient time steps. The generator duct

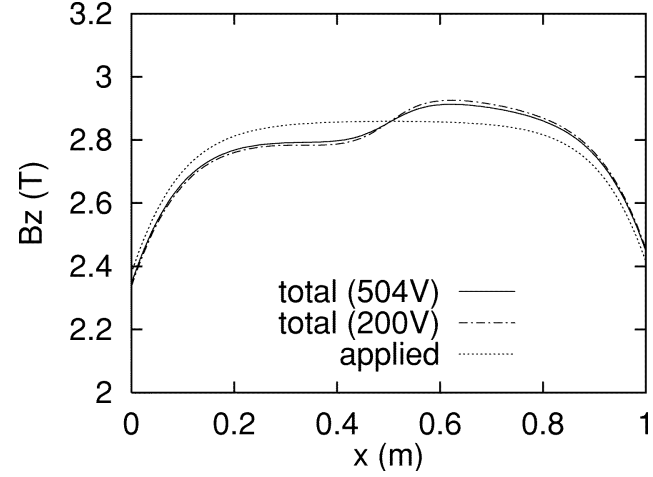

(a)

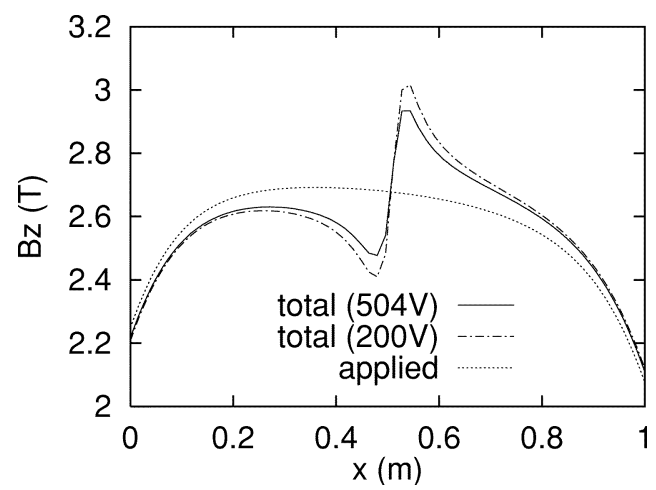

(b)

Fig. 2. Distribution of $B_{z}(z=0)$; (a) along the channel center $(y=0)$ and (b) along the anode electrode.

is divided into three subdomains according to grid size, which correspond to the 1) boundary layer, 2) core-flow region, and 3) intermediate region between 1) and 2). These three subdomains have the time steps of $31.25,125$, and $500 \mathrm{~ns}$, respectively.

The terms $\boldsymbol{J} \times \boldsymbol{B}$ and $\boldsymbol{J} \cdot \boldsymbol{E}$ in (14) and (15) are determined from the electromagnetic field analysis as described in the preceding subsection. The electromagnetic field is updated every 1 $\mu \mathrm{s}$, where $B_{i 0}$ is set at the value of $B_{i}$ at the previous time step.

\section{Simulation Results}

\section{A. Simulation of Maximum Output Operation}

First, an MHD field is analyzed to reconstruct the experimental results at hot-fire test no. 5 that achieved the maximum power output of Pamir-3U [2]. The inlet stagnation pressure and temperature are set at $46 \mathrm{~atm}$ and $3880 \mathrm{~K}$, respectively. The channel voltage is $504 \mathrm{~V}$. The applied magnetic flux density has the distribution of $B_{z}$ shown by the dotted lines in Fig. 2. It is assumed that the electrode currents are taken through a pair of current leads along the $y$ direction from the centers of the electrodes.

Figs. 2,,-5 depict distributions of steady-state MHD field in the power-generating region $(0<x<1 \mathrm{~m})$. Fig. 2 shows the distribution of $B_{z}$ along the flow direction at $z=0 \mathrm{~m}$. The solid lines in Fig. 2(a) and (b) show the distributions along the channel center $(y=0 \mathrm{~m})$ and along the anode electrode, respectively. Figs. 3-5 depicts the distributions of electric current density, gas velocity, and static pressure, respectively. The channel current computed is $19.5 \mathrm{kA}$, which roughly agrees with the experimental data $(20.8 \mathrm{kA})$. 


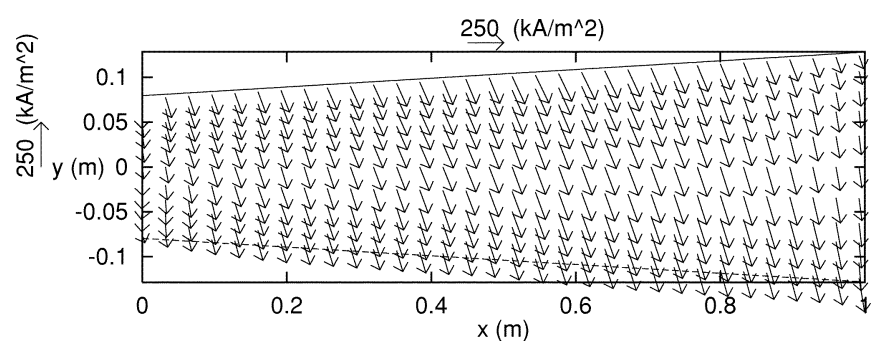

(a)

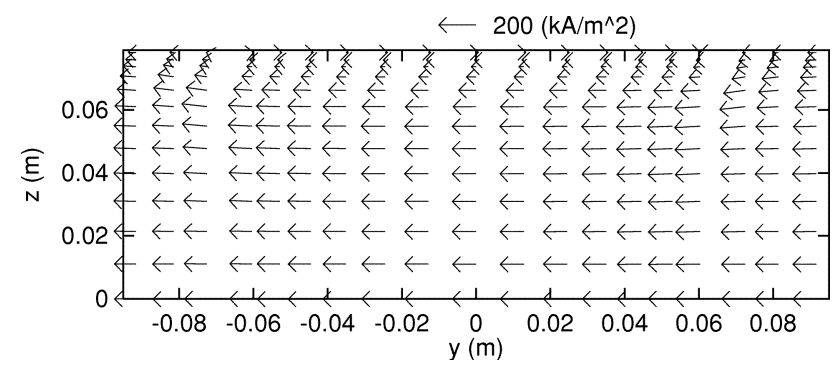

(b)

Fig. 3. Distributions of electric current density - channel voltage $504 \mathrm{~V}$. (a) Distribution of $\left(J_{x}, J_{y}\right)$ at $z=0 \mathrm{~m}$. (b) Distribution of $\left(J_{y}, J_{z}\right)$ at $x=0.32 \mathrm{~m}$.

Fig. 2(b) shows that the induced magnetic field becomes about $10 \%$ of the applied field near the electrode center. This is because the electrode current concentrates at the electrode center in order to be taken out through the current lead along the $y$ direction.

Fig. 3(a) shows that not only a Faraday current $\left(J_{y}\right)$ but also a Hall current $\left(J_{x}\right)$ is generated, which is due to the Hall electric field $\left(E_{x}\right)$ induced by $J_{y}$. A reverse current flow toward the anode electrode is seen along the insulator wall $(z=0.079 \mathrm{~m})$ in Fig. 3(b) because the electromotive force $(\boldsymbol{u} \times \boldsymbol{B})$ becomes small in the boundary-layer region.

Fig. 4(a) depicts that the flow velocity distribution is almost symmetric along the Faraday direction. Fig. 4(b) shows that the distribution of $u_{y}$ and $u_{z}$ is also almost symmetric in the upstream region, whereas the distribution becomes asymmetric in the downstream region, as shown in Fig. 4(c). The flow toward both electrode walls in Fig. 4(b) is caused by the duct shape with divergent walls. Fig. 4(c) shows that the plasma flow toward the cathode electrode occurs in the core-flow region (near $z=0 \mathrm{~m}$ ), whereas the flow toward the anode electrode is seen along the insulator wall. This asymmetric flow (secondary flow) is caused by the Hall current of which the Lorentz force pushes the core flow toward the cathode side.

The flow pushed toward the cathode side results in a higher pressure in the cathode side than in the anode side, as shown in Fig. 5. The pressure fluctuation in Fig. 5 is caused by an oblique shock wave due to the change of the duct angle at $x=0 \mathrm{~m}$.

\section{B. MHD Field With Excessive Electrode Current}

Experimental and numerical studies [2], [5] have shown that the excessive channel current reduces the power output because of the boundary-layer separation. Next, the channel voltage is decreased to $200 \mathrm{~V}$ in order to examine the effect of the large Lorentz force due to the large Faraday current.

Figs. 6-8 depict an obtained steady-state MHD field. A comparison of Fig. 6(a) with Fig. 3(a) shows that the decrease in the

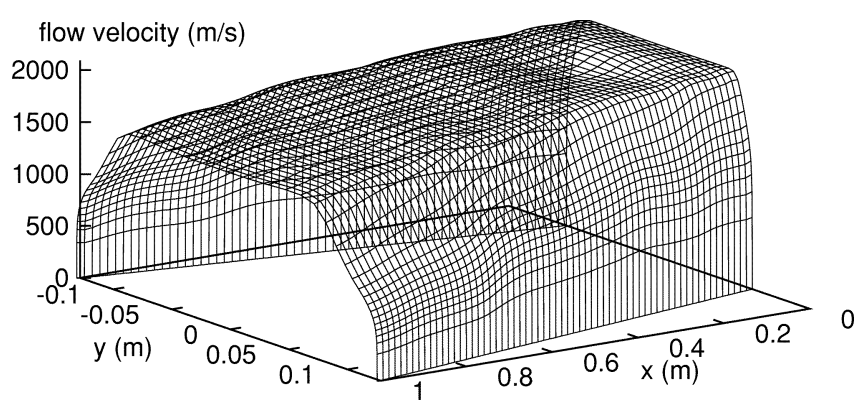

(a)

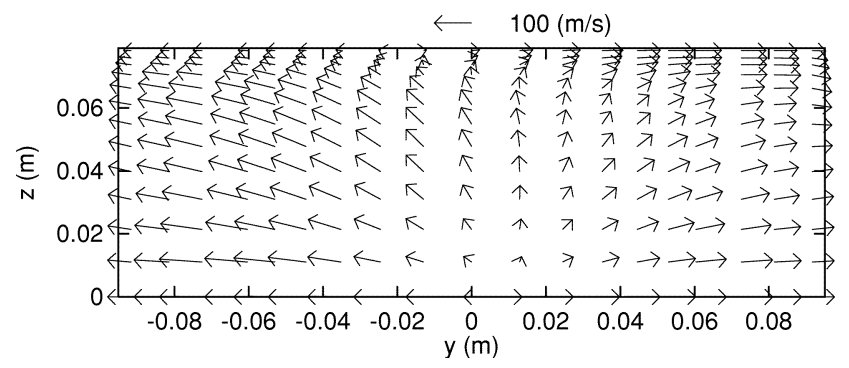

(b)

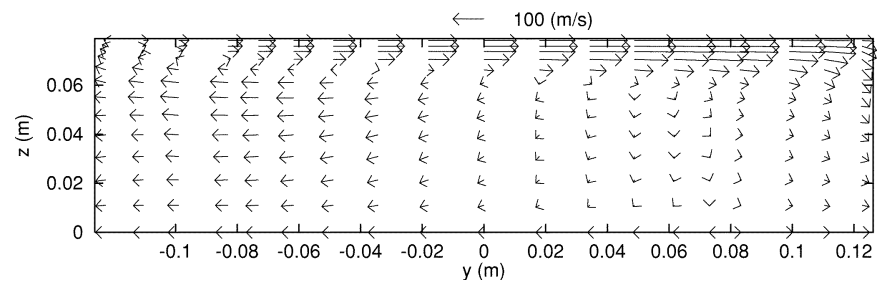

(c)

Fig. 4. Velocity distributions-channel voltage $504 \mathrm{~V}$. (a) $x-y$ distribution of $u_{x}$ at $z=0 \mathrm{~m}$. (b) Distribution of $\left(u_{y}, u_{z}\right)$ at $x=0.32 \mathrm{~m}$. (c) Distribution of $\left(u_{y}, u_{z}\right)$ at $x=0.96 \mathrm{~m}$.

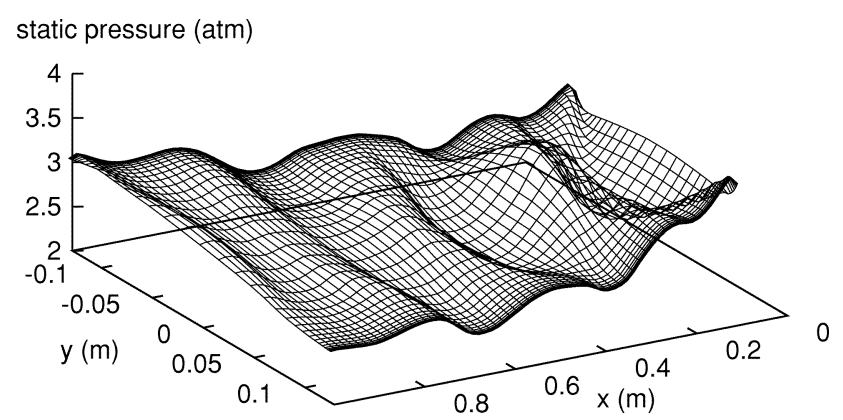

Fig. 5. Distribution of static pressure at $z=0 \mathrm{~m}$-channel voltage $504 \mathrm{~V}$.

channel voltage results in an increase in the Faraday current. A reverse current flow is seen near the insulator wall in Fig. 6(b). The sharp deceleration of the core-flow and the negative flow velocity in Fig. 7(a) depict, respectively, the shock wave and the boundary-layer separation, which are induced by the Lorentz force due to the large Faraday current. Fig. 7(b) shows that the boundary-layer separation induces a complex secondary flow, which has not been revealed by two-dimensional analyses [5], [7]. The sharp increase in static pressure in Fig. 8 is caused by the shock wave. The MHD field in Figs. 6-8 is asymmetric in the Faraday direction. This asymmetry is caused by the Hall current of which the Lorentz force pushes the working fluid toward the cathode side. 


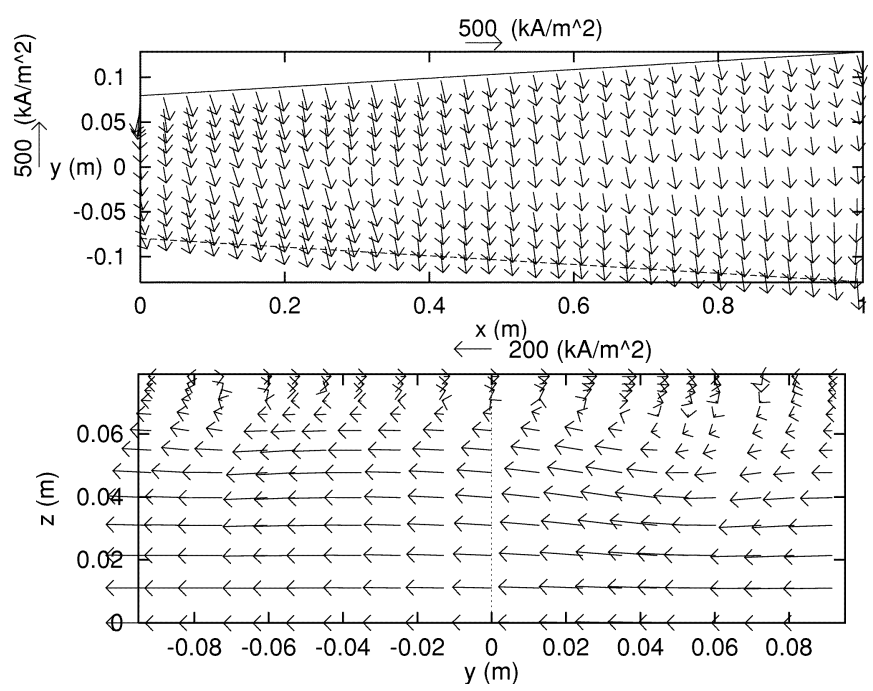

Fig. 6. Distributions of electric current density-channel voltage $200 \mathrm{~V}$. (a) Distribution of $\left(J_{x}, J_{y}\right)$ at $z=0 \mathrm{~m}$. (b) Distribution of $\left(J_{y}, J_{z}\right)$ at $x=0.32 \mathrm{~m}$.

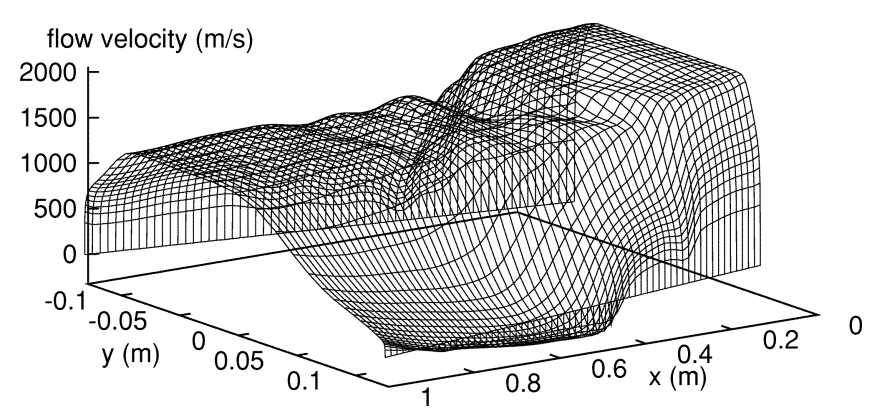

(a)

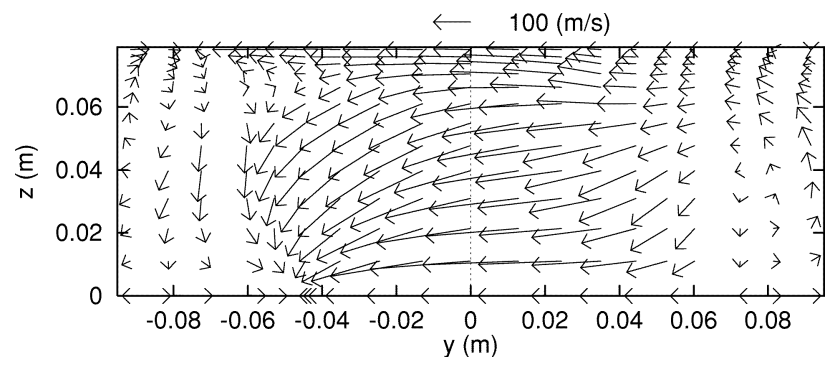

(b)

Fig. 7. Velocity distributions-channel voltage $200 \mathrm{~V}$. (a) $x-y$ distribution of $u_{x}$ at $z=0 \mathrm{~m}$. (b) Distribution of $\left(u_{y}, u_{z}\right)$ at $x=0.32 \mathrm{~m}$.

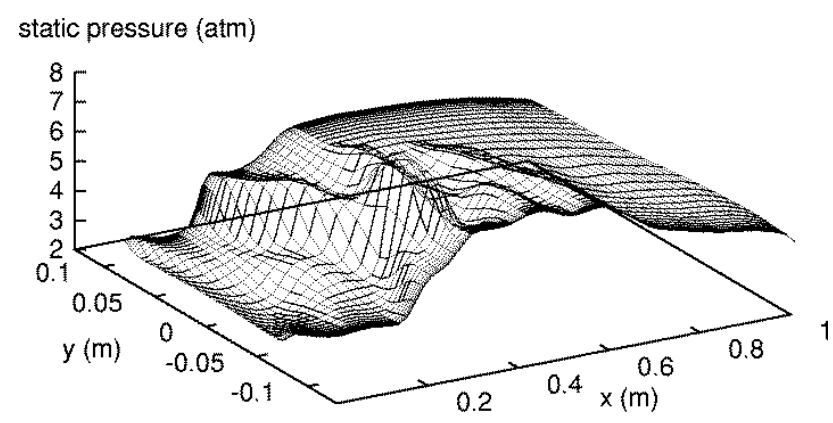

Fig. 8. Distribution of static pressure at $z=0 \mathrm{~m}$-channel voltage $200 \mathrm{~V}$.

The dash-dotted lines in Fig. 2 show the distribution of $B_{z}$, which shows that the induced magnetic field increases with the channel current.

\section{CONCLUSION}

A 3-D MHD field analysis taking into account the induced magnetic field is carried out for a supersonic plasma duct flow. The analysis reveals that the Lorentz force induces an asymmetric shock wave and boundary-layer separation in the plasma flow.

\section{ACKNOWLEDGMENT}

The authors would like to express their gratitude to Prof. M. Ishikawa, University of Tsukuba, Japan, for his advice on the analysis of the MHD generator.

\section{REFERENCES}

[1] V. A. Zeigarnik, "Pulsed MHD-generators," in Proc. 12th Int. Conf. MHD Power Generation, vol. 1, Yokohama, Japan, 1996, pp. 28-60.

[2] V. A. Zeigarnik et al., "The first experiments with pamir-3U pulsed portable MHD power system," in Proc. 33rd Symp. Engineering Aspects MHD, 1995, pp. I.5 1-11.

[3] B. S. Baldwin and H. Lomax, "Thin layer approximation and algebraic model for separated turbulent flows," AIAA, Paper 78-257, 1978.

[4] H. W. Stock and W. Hasse, "The determination of turbulent length scale in algebraic turbulence model for attached and slightly separated flows using Navier-Stokes methods," AIAA, Paper 87-1302, 1987.

[5] T. Matsuo, H. Sugita, M. Ishikawa, and V. A. Zeigarnik, "Numerical analysis of asymmetric strong shock wave in pulsed MHD generator," in Proc. 34th Symp. Engineering Aspects of MHD, 1997, pp. 3.1.1-3.1.10.

[6] H. C. Yee and A. Harten, "Implicit TVD schemes for hyperbolic conservation laws in curvilinear coordinates," AIAA J., vol. 25, no. 2, pp. 266-274, 1987.

[7] T. Matsuo and M. Shimasaki, "Parallel computation for high speed duct flow of weakly ionized plasma," in Proc. 4th Int. Conf. HPC-Asia 2000, vol. II, 2000, pp. 1075-1080. 